\title{
Long Bone Enchondroma vs. Low-Grade Chondrosarcoma: Current Concepts Review
}

\author{
Khodamorad Jamshidi, ${ }^{1}$ and Alireza Mirzaei ${ }^{1,{ }^{*}}$ \\ ${ }^{1}$ Bone and Joint Reconstruction Research Center, Shafa Orthopedic Hospital, Iran University of Medical Sciences, Tehran, Iran \\ "Corresponding author: Alireza Mirzaei, Shafa Orthopedic Hospital, Mojahedin Islam Avenue, Shohada Sq, Tehran, Iran. Tel: +98-2133542000-8, Fax: +98-2133542020, E-mail: \\ mirzaeialireza26@gmail.com
}

Received 2016 September 26; Accepted 2016 November 14.

\begin{abstract}
Context: Differentiation of low-grade chondrosarcoma (CS) from enchondroma (EC) is a clinical, radiological and pathological challenge. Considering its effect on the choice of therapeutic approach, this paper discusses the distinguishing criteria of low-grade CS and EC.

Evidence Acquisition: Evidence of this article came from the result of more than 15500 surgeries of musculoskeletal tumors performed at our center during the previous 26 years, in addition to an inclusive literature review of related published articles.

Results: Pain is considered as the most distinguishing clinical criteria of low-grade CS from EC. Aggressive radiologic criteria including cortical destruction, cortical thickening, deep endosteal scalloping and periosteal reaction favor the presence of low-grade CS. Predominantly, intermediate signal on T1-weighted MRI, multilocular appearance on contrast-enhanced T1-weighted MRI, extensive gadolinium enhancement, and peri-tumoral edema could also favor low-grade CS. In addition, permeation of the bone marrow by cartilage and separation of cartilage lobules by fibrous bands could be regarded as the pathologic characteristics of low-grade CS. Conclusions: Radiological evaluation is considered as the first line method in the distinction of low-grade CS and EC. In this regard, attention should be paid to the presence of aggressive radiological features.
\end{abstract}

Keywords: Enchondroma, Low-Grade Chondrosarcoma, Differentiation Criteria

\section{Context}

Long-bone enchondroma (EC) is a benign cartilage lesion thought to arise from the growth plate. This cartilage remnant is subjected to the same growth factors as epiphyseal plate, and its growth stops in adulthood. Approximately $2 \%$ of the population has small cartilaginous islands in the medullary canal of their bone, presumably because of this mechanism (1). Walden et al. identified $2.9 \%$ incidental enchondroma in 449 consecutive routine knee MR examinations. The highest incidence was in distal femur (2.0\%), proximal tibia (0.7\%) and proximal fibula $(0.2 \%)$. Most lesions were located in the metaphysis and the size of the lesion was less than $1 \mathrm{~cm}$ in the majority of cases (2).

Chondrosarcoma (CS), accounting for 10 to $20 \%$ of all primary bone malignancies, is the most common primary bone tumor of adults aged more than 25 years old, representing a wide spectrum of diseases $(3,4)$. The most common form of long-bone CS is central CS of long bone (conventional variety) (3). In terms of histology, CSs are differentiated into grade 1 (low-grade), grade 2 (intermediate grade) and grade 3 (high-grade), considering the cellularity, cellular atypia and mitosis. About $75 \%$ of central CSs are low grade (5). Low-grade CS was renamed atypi- cal cartilaginous tumors (ACT) by the world health organization in 2013 (6). Dedifferentiated CS is a highly malignant variant of CS, characterized by the development of a high-grade, non-cartilaginous sarcoma, in association with a pre-existing low-grade CS (7).

Diagnosis of bone tumors is generally based on clinical, radiological and pathological findings. Although radiological and histological characteristics allow the differentiation of high-grade CSs from EC, distinguishing lowgrade CS form EC is often difficult and sometimes impossible (8-10). However, this differentiation is of critical importance when it comes to the choice of therapeutic strategy. Enchondroma only requires follow up and no aggressive approach is indicated considering its benign behavior, whereas low-grade CS needs surgical treatment. The goal of this manuscript was to provide an overview of these two lesions, considering their clinical symptoms, radiological criteria and pathologic features.

\section{Evidence Acquisition}

During 26 years, more than 15500 surgeries were performed for patients with musculoskeletal tumors at the tumor surgery department of Shafa orthopedic hospital, 
which is the main referral center of our country. Considering these comprehensive experiences, we performed an inclusive literature review of related papers published in the most prestigious journals and tumor surgery textbooks to define the most accurate clinical, radiological and histological criteria for the differentiation of EC from low-grade CS.

\section{Results}

\subsection{Clinical Differences}

Long-bone EC is formed by entrapment of a portion of epiphyseal plate in the metaphysis during bone growth, which cease to grow in adulthood. Consequently, this lack of growth of the lesion does not cause pain (11). It is usually found incidentally on radiograph, bone scan or MRI during work-ups for other reasons. For instance, a middleaged patient, who had non-tumor related shoulder or knee pain, had a cartilage lesion found in the metaphysis during a radiographic study (Figure 1).

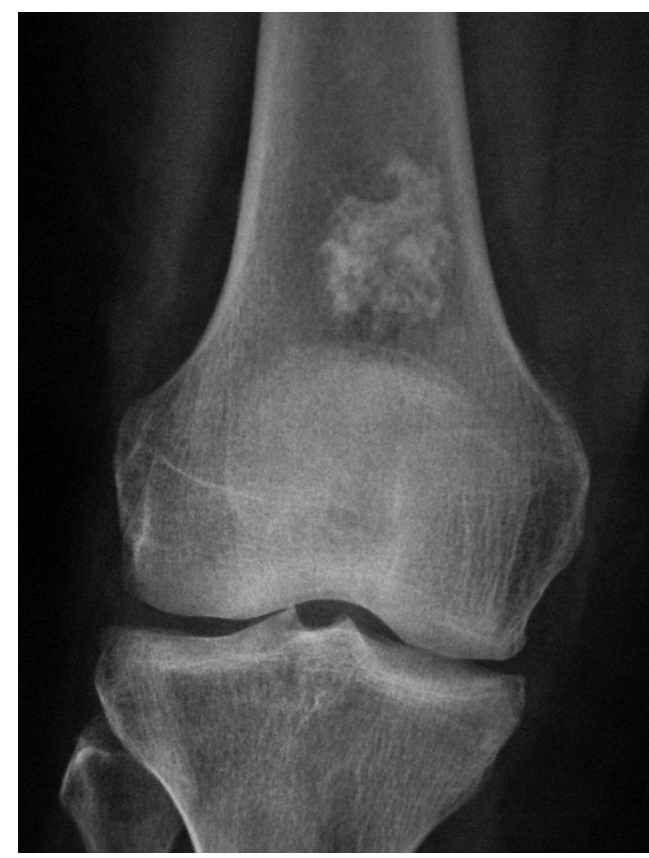

Figure 1. An Enchondroma Incidentally Noticed in the Distal Femur Radiograph of a 49-Year-Old Woman Who Presented Knee Pain Due to Early Degenerative Joint Disease of Knee

Another example is when aTc99 bone scan is used to detect bone metastasis in an already diagnosed carcinoma, and an increased uptake is spotted in proximal humerus or distal femur, where subsequent imaging indicates the presence of a cartilage lesion (Figure 2).
Rarely, EC may be complicated by stress fracture and the presence of functional pain (12), which makes the differentiation of EC from low-grade CS more complex. Enchondroma in short tubular bone of hands and feet grows and causes pain, as well.

In contrary, patients with a low-grade CS, experience tumor-related pain, which is caused by the growth of neoplasm. This pain is usually worse at night and is not relieved by rest or immobilization (11).

Bjornsson et al. also reported constant pain in $81 \%$ of long-bone primary CSs (13). However, the presence of pain only favors the diagnosis of low-grade CS and does not confirms it, as it has been reported in a considerable number $(34 \%)$ of ECs, as well $(8,14,15)$. In addition to pain, other clinical data including age and involved skeleton, could be employed in the differentiation of EC from low-grade CS. In this regard, cartilage lesions observed in patients older than 25 years old and in axial skeleton are more likely to be malignant $(10,16)$.

\subsection{Imaging Differences}

Tumor characteristics including margin, matrix, endosteal scalloping, cortical thickening, bone expansion, cortical breakthrough and size could be assessed through radiological evaluation of the lesion (12).

In younger patients, ECs are lytic lesions with welldefined margin in the central portion of metaphysis of metadiaphysis.

As patients get older, calcification and ossification of the normally radiolucent cartilage starts and radiodense ring and stipples appear (Figure 3). Although mild endosteal scalloping might be present, cortical erosion and thickening is not present in ECs (11).

Occasionally, the mineralization can be very dense but it is well defined without sclerotic border (Figure 4).

Low-grade CSs are usually more voluminous than ECs. Geirnaerdt et al. set a cutoff of $5 \mathrm{~cm}$ to distinguish between EC and low-grade CS (14), whereas Kendell et al. proposed a 4-cm limit (17). However, it is important to mention that a CS is small at the beginning and large benign lesions may also be found, making the size an inconsistent discriminator of EC and low-grade CS.

Low-grade CSs also demonstrate ring shaped and stippled mineralization of cartilage. However, unlike sharply circumscribed mineralization of ECs, a continuous line cannot trace CSs' mineralization. Deep endosteal scalloping also favors the diagnosis of low-grade CS. Furthermore, one or both cortices are often expanded in CSs, which is a manifestation of neoplastic growth. Cortical erosion, periosteal reaction and cortical thickening may also occur as a result of tumor invasion to haversian system (Figure 5). 

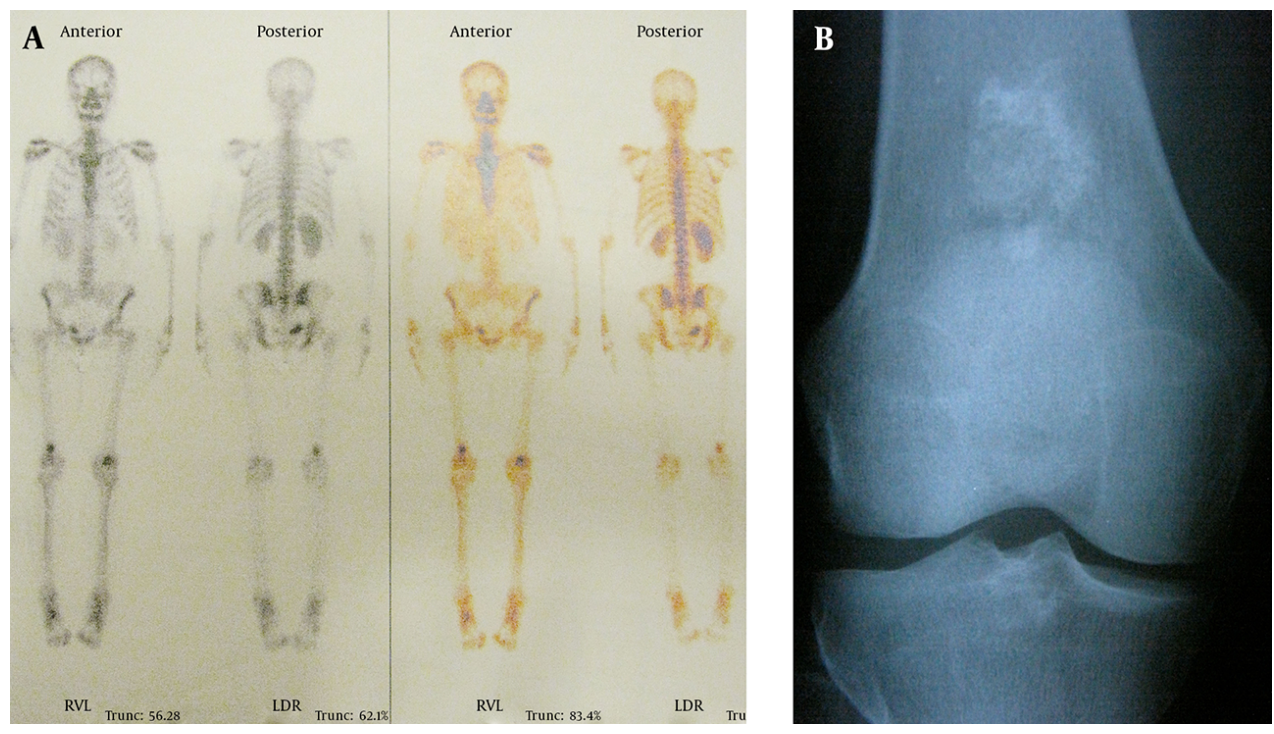

Figure 2. (A) Bone scan of a 41-year-old woman with two years. history of breast cancer, indicating an increased uptake equal to the iliac crest in the distal femur; (B) Distal femur Radiograph showed an EC.

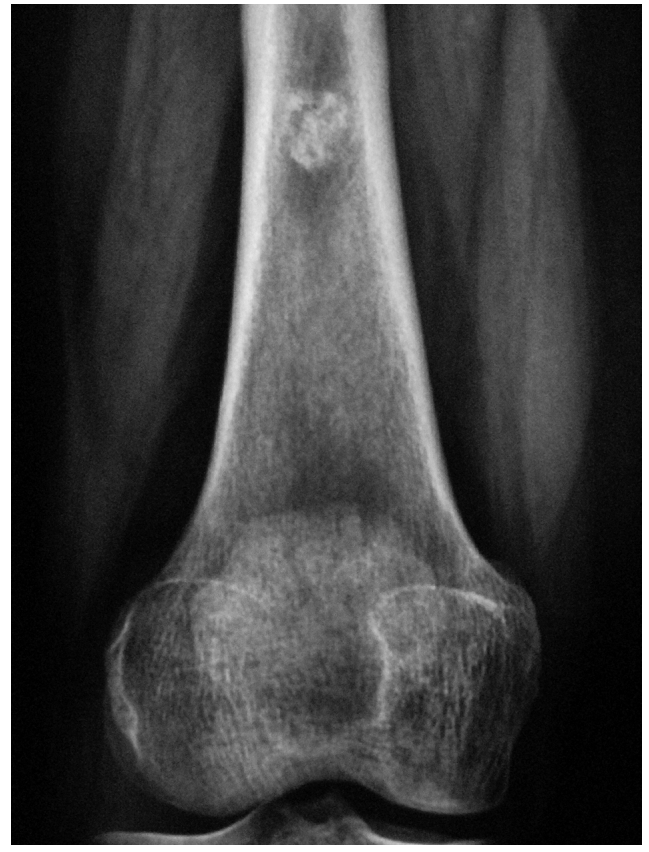

Figure 3. Presence of an Enchondroma in the Metaphysis of Femur of a 36-Year-Old Woman

Murphey et al. found that endosteal scalloping depth in the CT-scan was particularly discriminative. According to their report, $90 \%$ of CSs and only $10 \%$ of ECs demonstrated a scalloping of more than two-thirds of the cortical

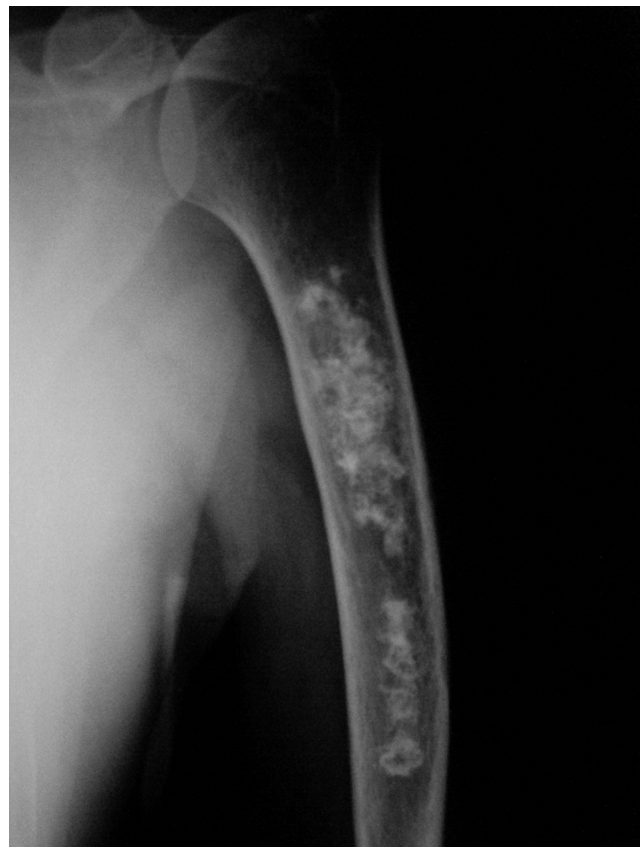

Figure 4. A 60-Year-Old Lady with Shoulder Pain Related to Painful Arc Syndrome. Radiograph Showed a Dense Well-Defined Catilaginous Lesion (EC).

thickness (8). However, it should be noted that all grades of CSs and not only grade I were included in their study. Geirnaerdt et al. reported a 90\% likelihood of CS when four criteria of CS, including ill-defined margins, lobulated con- 


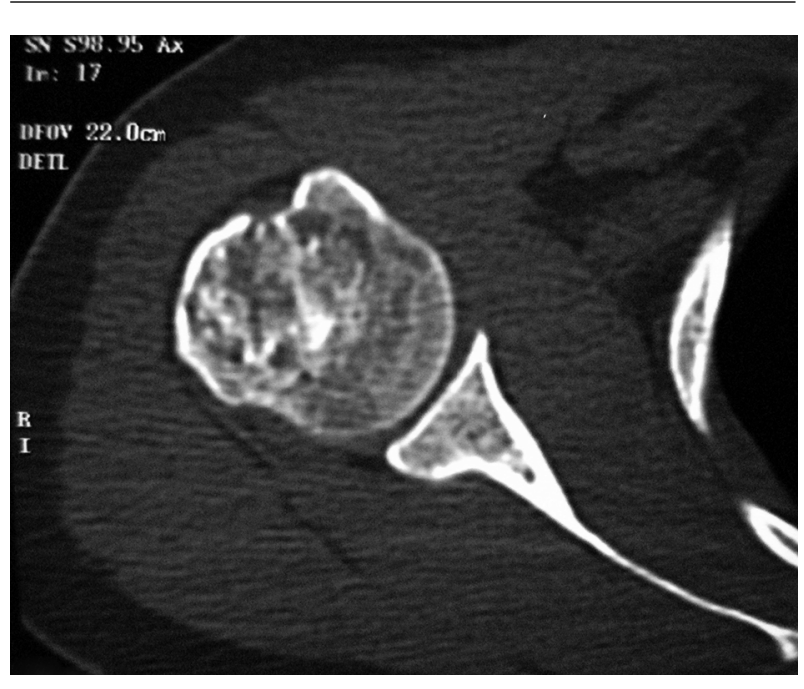

Figure 5. Computerized-Tomography Scan Indicating Cortical Erosion in a LowGrade Chondrosarcoma

tours, pop-corn calcifications and endosteal scalloping are present. However, this combination was only observed in $6 \%$ of their study population (14).

Occasionally, low-grade CS may cause a soft-tissue mass, which is never seen in EC (11). Parlier-Cuau reported $16 \%$ of soft-tissue mass in grade I CSs (9).

\subsubsection{Magnetic Resonance Imaging (MRI)}

The role of MRI is mostly to determine the extent of the lesion, because the main questions are already answered upon a radiograph. Choi et al. reported that patients with low-grade CSs had a significantly higher incidence of MR findings including: predominantly intermediate signal on T1-weighted images (Figure 6A), multilocular appearance on contrast-enhanced T1-weighted images, cortical erosion/destruction, peri-tumoral edema and an involvement of the epiphysis or flat bone. They concluded that MR imaging shows helpful features for differentiating low-grade CS from EC (18). Extensive gadolinium enhancement (Figure 6B) could also favor the presence of low-grade CS, while peripheral gadolinium enhancement favors EC (19).

\subsubsection{Bone Scintigraphy Differences}

Radionuclide uptake is present in both EC and lowgrade CS. However, the level of this uptake may be different. Bone scintigraphy could be employed in this distinction. Murphey et al. proposed to compare the radionuclide uptake of the tumor to the uptake of the anterior iliac crest. They found a superior radionuclide uptake in $82 \%$ of CSs, while lower or equal to that of anterior iliac crest in $79 \%$ of
ECs (8). Again, it should be noted that in Murphey et al.'s study all grades of CSs were included. In this regard, FerrerSantacreu et al. compared the radionuclide uptake of ECs with low-grade CSs. Their study showed no significant difference in radionuclide uptake of these two entities (10). Figure 7 shows higher radionuclide uptake of a low-grade CS compared to the uptake of the anterior iliac crest, while it is equal or lower in EC (Figure 2A).

\subsubsection{Histological Differences}

Enchondromas consist of mature hyaline cartilage lobules of varying cellularity. These cellular ECs often contain chondrocytes with plump or double nuclei. Occasionally, focal necrosis or myxoid change is present (11). Two important low-power characteristics of ECs reflect the lack of cartilage growth. First, cartilage lobules are usually separated by normal marrow. Second, cartilage lobules are partially encased by mature lamellar bone, which reflects prior enchondral ossification at the periphery of the lobule (20).

There is a considerable overlap in the histological features of EC and CS, and low-grade CSs usually cannot be distinguished by microscopic studies alone. Indeed, some CSs have areas of pre-existing EC. The cartilage of low-grade CS is cellular and plump binucleated chondrocytes are frequently observed. Despite these shared characteristics of EC and CS, there is one histological feature, which differentiates CS from EC. Permeation of the bone marrow by cartilage, which indicates neoplastic growth, is only present in CS. Another histological characteristic of CS is separation of cartilage lobules by fibrous bands, while in EC, lobules are separated by bone marrow or encased by bone. Furthermore, CSs may infiltrate the harversion canals of the cortex to form a soft-tissue mass. In that case, differentiation of EC and CS would be much less difficult (21). Extensive myxoid changes or areas of fibrosis are also in favor of CS (Figure 8).

Immunohistochemistry could also be employed in the distinction of growing versus non-growing lesions. In this regard, Ki-67, a protein, which is synthesized in the nuclei of replicating cells, could reflect the presence of a growing lesion. Positive Ki-67, even if only in a few cells, indicates that the lesion is growing, while no nuclei are stained with Ki-67 in non-growing lesions such as ECs (11).

\section{Conclusions}

The radiological evaluation is considered as the first line in determination of a management approach. Presence of aggressive criteria including cortical destruction, cortical thickening, periosteal reaction, deep endosteal scalloping, predominantly intermediate signal on T1-weighted MRI, multilocular appearance on 

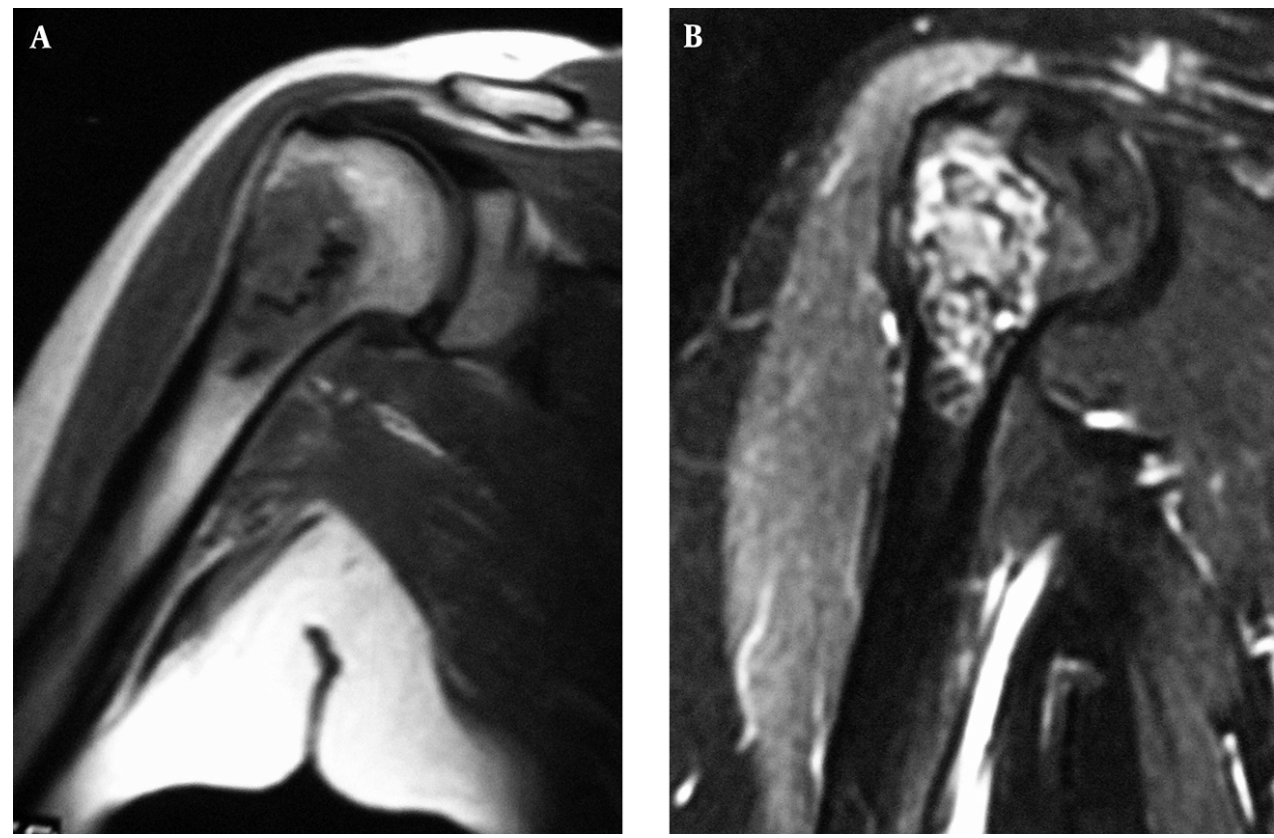

Figure 6. (A) Presence of predominantly intermediate signal on T1-weighted Magnetic Resonance Imaging of low-grade Chondrosarcoma(CS) lesion;(B) Extensive gadolinium enhancement of a low-grade CS.
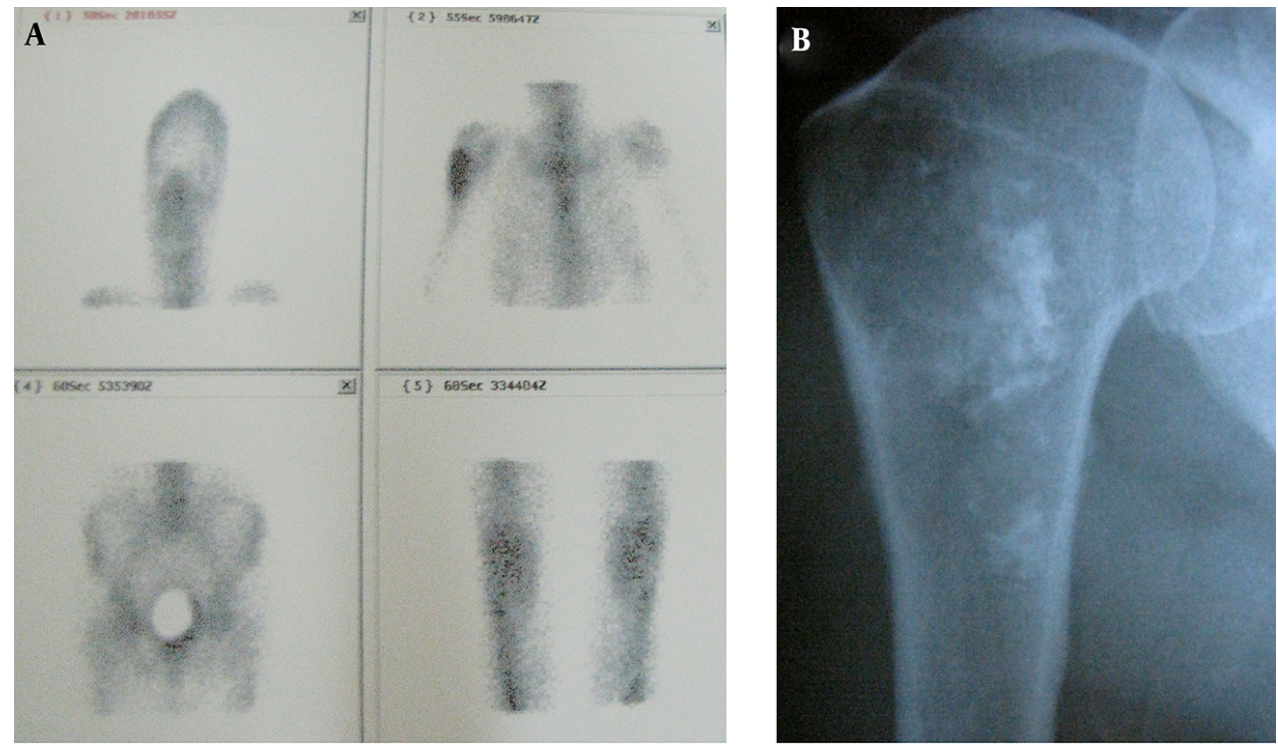

Figure 7. (A) Bone scan of a cartilaginous lesion showing higher uptake of tumor compared to iliac crest; (B) Corresponding radiograph indicating cartilaginous lesion diagnosed as low-grade CS.

contrast-enhanced T1-weighted MRI, extensive gadolinium enhancement, and peri-tumoral edema could favor the diagnosis of low-grade CS. Tumor related pain could also be considered as the clinical criterion of low-grade CS. Interpretation of pathologic findings could also help the clinical and radiological differentiation. 

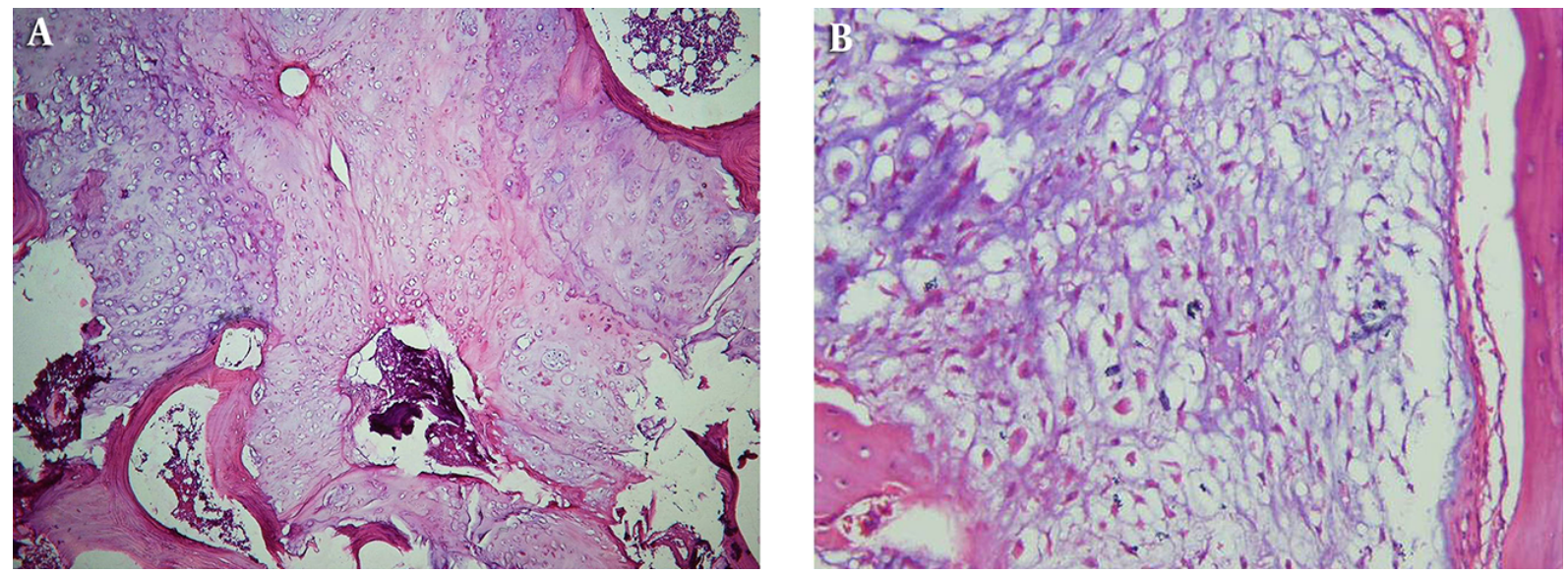

Figure 8. (A) Cartilage lobules partially encased by mature lamellar bone in an Enchondroma pathologic slide (H\&E 40X); (B). Cellular cartilage and plump binucleated chondrocytes embracing mature bone and separation of cartilage lobules by fibrous bands in a low-grade CS pathologic slide (H\&E 100X).

\section{References}

1. Scherer E. Exostosen, enchondrome und ihre beziehung zum periost. Frankfurt Ztschr F Path. 1928;36:587-605.

2. Walden MJ, Murphey MD, Vidal JA. Incidental enchondromas of the knee.AJR Am Joentgenol. 2008;190(6):1611-5. doi:10.2214/AJR.07.2796. [PubMed: 18492914].

3. Henderson ED, Dahlin DC. Chondrosarcoma of Bone-a Study of Two Hundred and Eighty-Eight Cases. J Bone Joint Surg Am. 1963;45:1450-8. [PubMed: 14069783].

4. Rosenthal DI, Schiller AL, Mankin HJ. Chondrosarcoma: correlation of radiological and histological grade. Radiology. 1984;150(1):21-6. doi: 10.1148/radiology.150.1.6689763. [PubMed: 6689763].

5. Dorfman H. Chondrosarcoma variants. Radiology Today: Springer; 1983. pp. $165-70$

6. Fletcher CDM, World Health O.. WHO classification of tumours of soft tissue and bone:[this book reflects the views of a working group that convened for a consensus and editorial meeting at the University of Zurich, Switzerland, 18-20 April 2012]. Internat. Agency for Research on Cancer; 2013.

7. Staals EL, Bacchini P, Bertoni F. Dedifferentiated central chondrosarcoma. Cancer. 2006;106(12):2682-91. doi: 10.1002/cncr.21936. [PubMed: 16691621].

8. Murphey MD, Flemming DJ, Boyea SR, Bojescul JA, Sweet DE, Temple HT. Enchondroma versus chondrosarcoma in the appendicular skeleton: differentiating features. Radiographics. 1998;18(5):1213-37. doi: 10.1148/radiographics.18.5.9747616. [PubMed: 9747616] quiz 1244-5.

9. Parlier-Cuau C, Bousson V, Ogilvie CM, Lackman RD, Laredo JD. When should we biopsy a solitary central cartilaginous tumor of long bones? Literature review and management proposal. Eur J Radiol. 2011;77(1):6-12. doi: 10.1016/j.ejrad.2010.06.051. [PubMed: 21241899].

10. Ferrer-Santacreu EM, Ortiz-Cruz EJ, Gonzalez-Lopez JM, Perez Fernandez E. Enchondroma versus Low-Grade Chondrosarcoma in Appendicular Skeleton: Clinical and Radiological Criteria. J Oncol. 2012;2012:437958. doi: 10.1155/2012/437958. [PubMed: 22593766].

11. McCarthy EF, Tyler WK. Distinguishing enchondroma from low-grade central chondrosarcoma. Pathol Case Reviews. 2001;6(1):8-13. doi:
10.1097/00132583-200101000-00003.

12. Mirra JM, Gold RH, Marcove RC. Bone tumors, diagnosis and treatment: Diagnosis and treatment. Lippincott Williams \& Wilkins; 1980.

13. Björnsson J, McLeod RA, Unni KK, Ilstrup DM, Pritchard DJ. Primary chondrosarcoma of long bones and limb girdles. Cancer. 1998;83(10):2105-19.

14. Geirnaerdt MJ, Hermans J, Bloem JL, Kroon HM, Pope TL, Taminiau $\mathrm{AH}$, et al. Usefulness of radiography in differentiating enchondroma from central grade 1 chondrosarcoma. AJR Am J Roentgenol. 1997;169(4):1097-104. doi: 10.2214/ajr.169.4.9308471. [PubMed: 9308471].

15. Potter BK, Freedman BA, Lehman RJ, Shawen SB, Kuklo TR, Murphey MD. Solitary epiphyseal enchondromas. J Bone Joint Surg Am. 2005;87(7):1551-60. doi: 10.2106/JBJS.D.02404. [PubMed: 15995123].

16. Eefting D, Schrage YM, Geirnaerdt MI, Le Cessie S, Taminiau AH, Bovee $\mathrm{JV}$, et al. Assessment of interobserver variability and histologic parameters to improve reliability in classification and grading of central cartilaginous tumors. Am J Surg Pathol. 2009;33(1):50-7. doi 10.1097/PAS.0b013e31817eec2b. [PubMed: 18852676].

17. Kendell SD, Collins MS, Adkins MC, Sundaram M, Unni KK. Radiographic differentiation of enchondroma from low-grade chondrosarcoma in the fibula. Skeletal Radiol. 2004;33(8):458-66. doi: 10.1007/s00256-004-0791-9. [PubMed: 15221215].

18. Choi BB, Jee WH, Sunwoo HJ, Cho JH, Kim JY, Chun KA, et al. MR differentiation of low-grade chondrosarcoma from enchondroma. Clin Imaging. 2013;37(3):542-7. doi: 10.1016/j.clinimag.2012.08.006. [PubMed: 23041161].

19. Crim J, Schmidt R, Layfield L, Hanrahan C, Manaster BJ. Can imaging criteria distinguish enchondroma from grade 1 chondrosarcoma? Eur J Radiol. 2015;84(11):2222-30. doi: 10.1016/j.ejrad.2015.06.033. [PubMed: 26220916].

20. Mirra JM, Gold R, Downs J, Eckardt IJ. A new histologic approach to the differentiation of enchondroma and chondrosarcoma of the bones. A clinicopathologic analysis of 51 cases. Clin Orthop Relat Res. 1985(201):214-37. [PubMed: 4064409].

21. Schiller AL. Diagnosis of borderline cartilage lesions of bone. Semin Diagn Pathol. 1985;2(1):42-62. [PubMed: 3870763]. 